\title{
First-in-Man Clinical Pilot Study Showing the Safety and Efficacy of Intramuscular Injection of Basic Fibroblast Growth Factor With Atelocollagen Solution for Critical Limb Ischemia
}

\author{
Kazunori Ono, MD; Kenji Yanishi, MD, PhD; Makoto Ariyoshi, MD, PhD; \\ Satoshi Kaimoto, MD, PhD; Motoki Uchihashi, MD, PhD; \\ Keisuke Shoji, MD; Satoaki Matoba, MD, PhD
}

\begin{abstract}
Background: Therapeutic angiogenesis with basic fibroblast growth factor (bFGF) with atelocollagen was confirmed in a study using a limb ischemia mouse model. Because the number of elderly patients with critical limb ischemia (CLI) is increasing, particularly that caused by arteriosclerosis obliterans (ASO), the development of less invasive angiogenesis therapies desired.

Methods and Results: This first-in-man clinical study was designed to assess the safety and efficacy of i.m. injection of bFGF with atelocollagen. Human recombinant bFGF $(200 \mu \mathrm{g})$, combined with $4.8 \mathrm{~mL} 3 \%$ atelocollagen solution, was prepared and injected into the gastrocnemius muscle of the ischemic leg. The primary endpoint was safety, evaluated on all adverse events over 48 weeks after this treatment. The secondary endpoint was efficacy, evaluated by improvement of ischemic symptoms. No serious procedurerelated adverse events were observed during the follow-up period. Visual analogue scale (VAS) score was significantly improved at 4, 24 and 48 weeks compared with baseline $(\mathrm{P}<0.05)$, and 7 patients became pain free during the follow-up period. Fontaine classification was improved in 4 of 10 patients at 48 weeks. Cyanotic lesions disappeared in 2 patients at 4 weeks.
\end{abstract}

Conclusions: I.m. injection of bFGF with atelocollagen is safe and feasible in patients with CLI. Randomized controlled trials are therefore needed to confirm these results.

Key Words: Basic fibroblast growth factor; Critical limb ischemia; Peripheral artery disease; Therapeutic angiogenesis

D ue to the recent rise in the number of diabetic patients, as a result of the aging of the general population worldwide, the prevalence of critical limb ischemia (CLI; Fontaine classification III and IV, or Rutherford classification categories 4, 5, and 6) has also been increasing. Although medical and surgical treatment, including percutaneous transluminal angioplasty (PTA) and bypass procedures, have markedly contributed to the treatment of CLI, many patients are forced to undergo amputation of the lower limbs because of arteriosclerosis obliterans (ASO) or Buerger's disease (thromboangiitis obliterans; TAO). In spite of the remarkable progress in the treatment of CLI, therapeutic outcomes remain far from satisfactory and the prognosis of CLI remains challenging. ${ }^{1-3}$ Therefore, new therapeutic approaches are needed for the effective management of CLI.

No effective alternatives to percutaneous or surgical revascularization presently exist for the treatment of CLI.
In recent years, cell therapies have been developed and are performed, in clinical practice, as advanced medical treatment. Procedures that facilitate angiogenesis and collateral circulation have recently been assessed for the reduction of tissue damage. These procedures include the use of growth factors (vascular endothelial growth factor, VEGF; hepatocyte growth factor, HGF; and basic fibroblast growth factor, bFGF and FGF-2), and bone marrow cells $\left(\mathrm{CD} 34^{+}\right.$or mononuclear cells $\left.[\mathrm{MNC}]\right) .^{49}$ The Therapeutic Angiogenesis by Cell Transplantation (TACT) trial reported on the safety and efficacy of i.m. implantation of bone marrow MNC (BM-MNC implantation) as a treatment for CLI, and, in particular, those patients with ASO or TAO with no option for other treatment., ${ }^{\mathbf{8 1 0 , 1 1}}$ Concerns regarding the effectiveness, immune or inflammatory responses to genetic materials and invasiveness of gene therapy or cell transplantation, however, still exist. Many patients with CLI, especially those with ASO, have ischemic heart dis-

Received July 17, 2018; revised manuscript received September 19, 2018; accepted October 9, 2018; J-STAGE Advance Publication released online November 9, 2018 Time for primary review: 52 days

Department of Cardiovascular Medicine, Graduate School of Medical Science, Kyoto Prefectural University of Medicine, Kyoto, Japan

Mailing address: Kenji Yanishi, MD, PhD, Department of Cardiovascular Medicine, Graduate School of Medical Science, Kyoto Prefectural University of Medicine, 465 Kajii-cho Kawaramachi-Hirokoji, Kamigyo-ku, Kyoto 602-8566, Japan. E-mail: yanishi@koto.kpu-m.ac.jp

ISSN-1346-9843 All rights are reserved to the Japanese Circulation Society. For permissions, please e-mail: cj@j-circ.or.jp 
ease, cerebrovascular disease and lung disease. In addition, the number of elderly patients with ASO is increasing. Therefore, establishment of a less invasive angiogenesis treatment is desired.

Although treatments involving several growth factors have been assessed, direct injection of free growth factors failed to demonstrate efficacy in clinical trials. ${ }^{12}$ This lack of effectiveness was partially attributed to suboptimal drug delivery, which led to low levels of accumulation in lesions and to rapid inactivation. Given that growth factors have a short half-life and are unstable in vivo, angiogenic effects are not expected when growth factors are simply dissolved in water or injected i.m. ${ }^{\mathbf{1 3}, 14}$ Marui et al and Kumagai et al described the incorporation of bFGF into a gelatin hydrogel as a novel approach to enable sustained release of the growth factor. ${ }^{\mathbf{1 5 , 1 6}}$ The manufacturing cost of this hydrogel, however, prohibited its use in health care. Combination with atelocollagen was subsequently proposed as a more cost-effective method for the sustained release of bFGF. Atelocollagen has been widely used since 1986 as an intradermal filler to restore areas of defect in soft tissues. While this formulation presents as a viscous solution at room temperature, it turns into a gel form and remains at the treatment site at body temperature. Moreover, the gel is processed into low-antigenicity molecules and degraded slowly in vivo. ${ }^{17}$ The efficacy and safety of bFGF injection with an atelocollagen solution for promoting angiogenesis was confirmed in a mouse model of limb ischemia. ${ }^{18}$ Based on this evidence, the aim of this study was to perform a first human trial to evaluate the safety and efficacy of the bFGF-atelocollagen sustained release system in patients with CLI.

\section{Methods}

\section{Subjects}

Patients who met the following inclusion criteria were enrolled in the trial: (1) ASO or TAO; (2) Fontaine classification III-IV and Rutherford classification 4-6 in either limb; (3) no clinical improvement following conventional medical and surgical treatment; (4) intention to receive the protocol treatment for either limb; and (5) written informed consent. There was no limitation regarding age. In all cases, there was confirmation that patients were not candidates for surgical or non-surgical revascularization treatment of the limb ischemia by vascular surgeons, cardiologists and radiologists. The clinical diagnosis of TAO was based on the criteria by Olin or Shionoya. ${ }^{19,20}$

The exclusion criteria were as follows: (1) life expectancy $<1$ year; (2) scheduled major lower limb amputation; (3) history of angina, congestive heart failure or ventricular arrhythmia; (4) history of myocardial infarction in the last 3 months; (5) left ventricular ejection fraction $<25 \%$; (6) history of lower limb revascularization in the last 3 months; (7) history of percutaneous coronary intervention or bypass surgery in the last 1 month; (8) hemoglobin $\leq 7 \mathrm{~g} / \mathrm{dL}$; (9) platelet count $\leq 70,000 / \mu \mathrm{L}$; (10) aspartate aminotransferase $>100$ IU or alanine aminotransferase $\geq 100 \mathrm{IU}$; (11) present malignant neoplasm; (12) suspected febrile infection; (13) uncontrolled diabetes mellitus, defined as HbA1c $>10 \%$; (14) diabetic proliferating retinopathy; (15) not able to stop alprostadil alfadex (for injections), alprostadil (injection solution), argatroban (injection solution), or trafermin (recombinant) before injection; (16) history of severe drug allergies; (17) positive skin reaction before enrollment; (18) pregnancy or lactation; (19) history of alcohol or drug abuse; and (20) any other medical condition considered inappropriate for enrollment by the investigator.

The protocol was approved by the Ethics Review Board of the Kyoto Prefectural University of Medicine, Kyoto, Japan (approval no. C-572, 23 July 2009). Patients were registered via fax at the Translational Research Informatics (TRI) data center. This trial is registered at http://www. umin.ac.jp/ctr/index.htm (identifier: UMIN000005277).

\section{Preparation of bFGF With Atelocollagen}

A total of $250 \mu \mathrm{g}$ of human recombinant bFGF (FIBLAST ${ }^{\circledR}$ Spray; Kaken Pharmaceutical, Japan), in $1.0 \mathrm{~mL}$ distilled water, was prepared as the injection solvent and $6.0 \mathrm{~mL}$ ( $1.0 \mathrm{~mL} \times 6$ tubes) of a $3 \%$ atelocollagen solution (Koken Atelocollagen Implant ${ }^{\circledR}$; Koken, Japan) was prepared approximately $1 \mathrm{~h}$ prior to treatment. The bFGF solution was transferred into a clean 5-mL syringe and the atelocollagen solution was transferred into a clean $10-\mathrm{mL}$ syringe. Before the injection, the physician connected these syringes via a T-shape stopcock. The plunger was pushed slowly to administer the solution, and shuttled 50 times. In total, $4.8 \mathrm{~mL}$ of the solution was prepared $(120 \mu \mathrm{L}$ per site for 40 sites in one patient).

\section{Treatment}

Antiplatelet agents were discontinued 3-14 days before treatment. Warfarin was discontinued 7 days before treatment. The use of alprostadil, argatroban, and trafermin was prohibited starting 7 days before the protocol treatment until the end of the clinical trial. Other concomitant medications being used before registration into the trial, as well as medication that may affect blood flow in the lower limbs, were not changed during the study. Forty sites were marked on the gastrocnemius muscle of the ischemic leg prior to treatment. Under epidural anesthesia, $120 \mu \mathrm{L} \mathrm{bFGF}$ (FGF-2) with the atelocollagen solution was injected at each of the 40 sites per treatment, in one patient, using a $30-\mathrm{G}$ needle. The sites were selected based on a previous study by Marui et al. ${ }^{15}$

\section{Study Protocol (Primary and Secondary Endpoints)}

The i.m. injection of bFGF with the atelocollagen solution was carried out in patients with CLI satisfying the inclusion and exclusion criteria over a period of 2 years, from the start date of the trial. The primary endpoint was the safety of the injection measured at 48 weeks after treatment, assessed on all adverse events occurring during the follow-up period. Adverse events were evaluated in terms of their seriousness (serious or non-serious) and severity (mild, moderate and severe), and their relevance to the injection of bFGF with atelocollagen. The target limb events (injection site pain, severe infection and acute limb ischemia) and other minor events (such as allergy, peripheral neuropathy, visual impairment, and immune deficiency) were reported as the related adverse events of FIBLAST ${ }^{\circledR}$ Spray and Koken Atelocollagen Implant ${ }^{\circledR}$. Major amputation, overall survival, and major adverse cardiovascular events, such as death, acute myocardial infarction, heart failure, and stroke, were also assessed in the safety of this treatment.

The secondary endpoint was efficacy, as defined by improvements, from baseline, in ischemic pain at rest, 6-min walk distance, ankle-brachial index (ABI), Fontaine/ 


\begin{tabular}{|c|c|}
\hline & $\begin{array}{l}\text { All patients } \\
(n=10)\end{array}$ \\
\hline Age (years) & $73 \pm 15$ \\
\hline Age $>80$ years & 5 \\
\hline Male & 3 \\
\hline Height (cm) & $156 \pm 9.9$ \\
\hline Weight (kg) & $45 \pm 8.6$ \\
\hline BMI $\left(\mathrm{kg} / \mathrm{m}^{2}\right)$ & $18 \pm 2.2$ \\
\hline Hypertension & 9 \\
\hline Hyperlipidemia & 3 \\
\hline DM & 1 \\
\hline DM with insulin & 0 \\
\hline Hemodialysis & 0 \\
\hline Smoking & 1 \\
\hline History of major amputation & 2 \\
\hline \multicolumn{2}{|l|}{ Etiology } \\
\hline ASO & 9 \\
\hline TAO & 1 \\
\hline \multicolumn{2}{|l|}{ Complications } \\
\hline Ischemic heart disease & 2 \\
\hline Cerebrovascular disease & 1 \\
\hline Chronic kidney disease & 0 \\
\hline Fontaine classification (III/IV) & $5 / 5$ \\
\hline Rutherford Classification (4/5/6) & $5 / 5 / 0$ \\
\hline Target lower limbs (left/right) & $8 / 2$ \\
\hline \multicolumn{2}{|l|}{ Target limb condition } \\
\hline Presence of cyanosis & 2 \\
\hline$A B I$ & $0.8(0.8-1.0)^{\dagger}$ \\
\hline VAS $(\mathrm{cm})$ & $7.5(6.8-9.3)^{\dagger}$ \\
\hline 6-min walk distance $(\mathrm{m})$ & $60(8.8-118)^{\ddagger}$ \\
\hline \multicolumn{2}{|l|}{ Laboratory data } \\
\hline WBC $\left(\times 10^{3} / \mu \mathrm{L}\right)$ & $5.8(4.6-7.5)^{\dagger}$ \\
\hline $\mathrm{Ht}(\%)$ & $36(28-40)^{\dagger}$ \\
\hline $\mathrm{TP}(\mathrm{g} / \mathrm{dL})$ & $7.0(6.9-7.5)^{\dagger}$ \\
\hline $\mathrm{Cr}(\mathrm{mg} / \mathrm{dL})$ & $0.7(0.6-0.9)^{\dagger}$ \\
\hline CRP (mg/dL) & $0.8(0.3-1.0)^{\dagger}$ \\
\hline
\end{tabular}

Data presented as $n$, mean $\pm S D$, tmedian (IQR) or faverage (IQR). $\mathrm{ABI}$, ankle-brachial index; $\mathrm{ASO}$, arteriosclerosis obliterans; $\mathrm{Cr}$, creatinine; CRP, C-reactive protein; DM, diabetes mellitus; $\mathrm{Ht}$, hematocrit; TAO, thromboangiitis obliterans; TP, total protein; VAS, visual analogue scale; WBC, white blood cells.

Rutherford classification of CLI, ulcerous/necrotic lesions, and cyanosis at 2, 4, 8, 12, 24 and 48 weeks after i.m. injection of bFGF with atelocollagen. Ischemic pain at rest was evaluated using a visual analogue scale (VAS), with anchors at 0 (pain free) and at $10 \mathrm{~cm}$ (most severe pain).

\section{Statistical Analysis}

Continuous variables are presented as mean $\pm \mathrm{SD}$ or median (IQR), as appropriate for the data distribution, with categorical variables presented as number (count). Time-to-event analysis was performed using the Kaplan-Meier method to examine variables associated with overall survival (OS) and major amputation-free (MAF) rates. The VAS and 6-min walk distance at 4,24 or 48 weeks after treatment were compared with those at baseline using Wilcoxon signed-rank test. All statistical analysis was performed

\begin{tabular}{|c|c|}
\hline & $\begin{array}{c}\text { All patients } \\
(n=10)\end{array}$ \\
\hline All-cause death & 0 \\
\hline Major amputation & 0 \\
\hline Related adverse events & 0 \\
\hline Target limb events & 0 \\
\hline Injection site pain & 0 \\
\hline Severe infection & 0 \\
\hline Acute limb ischemia & 0 \\
\hline Others & 0 \\
\hline Allergy & 0 \\
\hline Peripheral neuropathy & 0 \\
\hline Muscle or joint pain & 0 \\
\hline Systemic infection & 0 \\
\hline Worsening of liver function & 0 \\
\hline \multicolumn{2}{|l|}{ No-related adverse events } \\
\hline Worsening of peripheral ischemia & 1 \\
\hline Levofloxacin-induced urticaria & 1 \\
\hline Insomnia-induced heart failure & 1 \\
\hline Drug eruption & 1 \\
\hline Nasopharyngitis & 1 \\
\hline Fundal hemorrhage & 1 \\
\hline Delirium & 1 \\
\hline Compression fractures & 1 \\
\hline \multicolumn{2}{|l|}{ MACE } \\
\hline Death & 0 \\
\hline Acute myocardial infarction & 0 \\
\hline Heart failure & 1 \\
\hline Stroke & 0 \\
\hline
\end{tabular}

Data given as n. MACE, major adverse cardiovascular events.

using IBM ${ }^{\circledR}$ SPSS $^{\circledR}$ version 23 (IBM, Armonk, NY, USA), with $\mathrm{P}<0.05$ considered to be statistically significant.

\section{Source Data Verification}

On source data verification of all patients recruited in this study, although informed consent was obtained from all patients before enrollment, 2 patients were registered after the treatment.

\section{Results}

\section{Subjects}

Table 1 lists the baseline and target lesion characteristics of all CLI patients $(n=10)$ enrolled in this study. The patients consisted of 3 men and 7 women, with an average age of 73 years, with 5 patients $>80$ years of age. The cause of CLI was ASO in 9 patients and TAO in 1 patient. Five patients had Rutherford classification 4, and 5 patients had Rutherford classification 5. Two patients could not walk at baseline due to a history of major amputation in the contralateral leg. Median ABI was 0.8 (IQR, 0.8-1.0), with a median VAS of $7.5 \mathrm{~cm}$ (IQR, 6.8-9.3) and 6-min walk distance of $60 \mathrm{~m}$ (IQR, 8.8-118) at baseline. Cyanosis of the target limb was identified in 2 patients (Table 1). Main baseline characteristics are listed separately per patient in Table S1. 


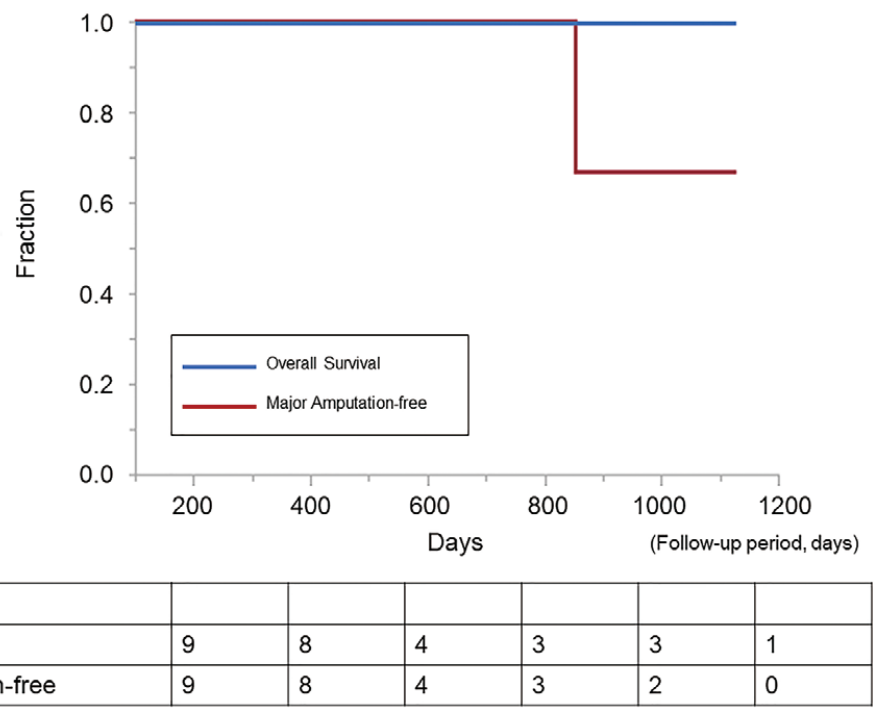

Figure 1. Kaplan-Meier analysis of overall survival and major amputation-free rates after i.m. injection of basic fibroblast growth factor with atelocollagen, in patients with critical limb ischemia.

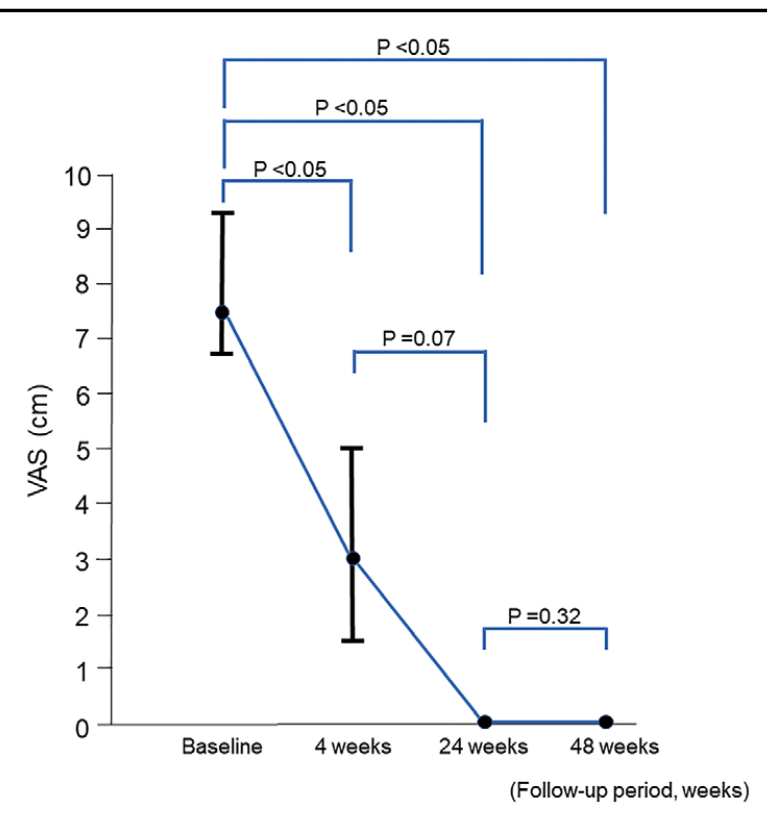

Figure 2. Change in visual analogue scale (VAS) score during the follow-up period, after i.m. injection of basic fibroblast growth factor with atelocollagen in patients with critical limb ischemia. Data given as median and IQR.

\section{Primary Endpoints}

The main adverse events after i.m. injection of bFGF with atelocollagen are listed in Table 2 . Three patients could not be followed up sufficiently during the period of observation: 1 patient due to comorbidity (compression fracture of the lumbar spine) at 301 days after the date of injection, and 2 patients due to socioeconomic reasons at 192 and 90 days. For all other patients, all events after i.m. injection of bFGF with atelocollagen were noted over the entire period of observation. The following adverse events were

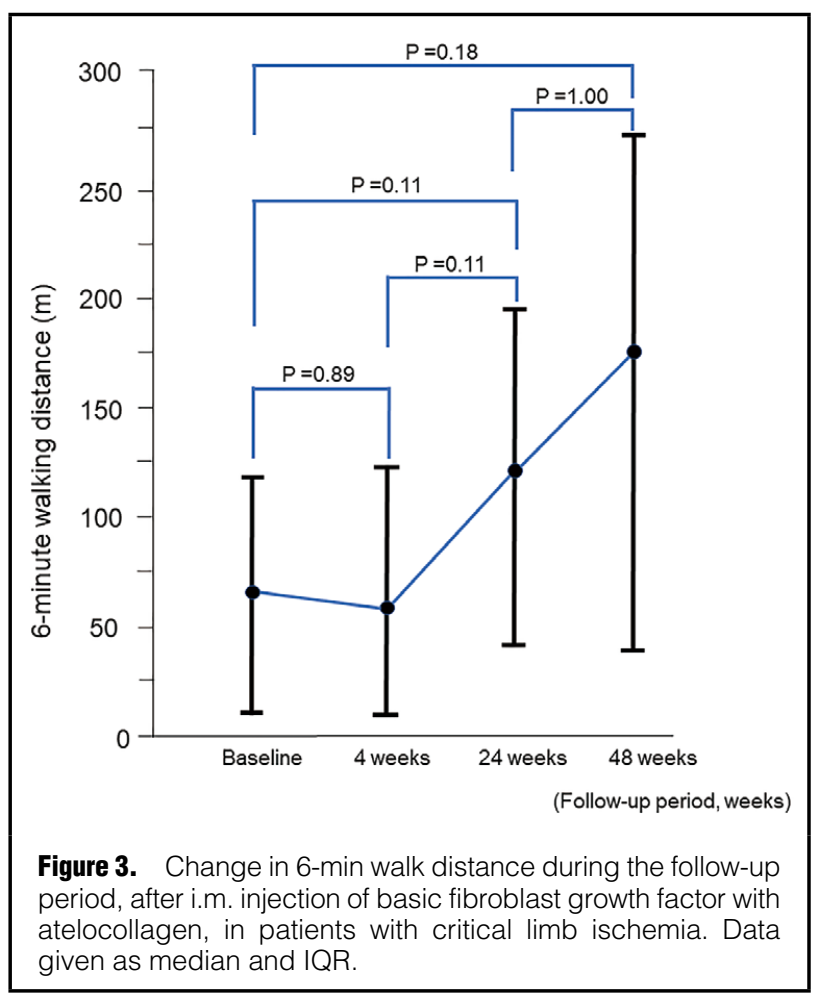

identified: peripheral ischemia, 1 patient; levofloxacininduced urticaria, 1 patient; and insomnia-induced heart failure, 1 patient. The following non-serious events were observed: 1 patient each had drug eruption caused by another drug; nasopharyngitis; fundal hemorrhage; and delirium; and 2 patients had compression fractures. All adverse events were graded as mild, and no patients died during the follow-up period. In addition, progression of peripheral ischemia due to worsening of the baseline disease occurred after 2 months of this procedure, but was 
improved in approximately 2 weeks by prostaglandin formulation. These adverse events were not related to the i.m. injection of bFGF with atelocollagen (Table 2). A minor increase in serum bFGF (up to $28 \mathrm{ng} / \mathrm{mL}$ ) was observed in 3 patients, but complications associated with this increase in serum bFGF were not confirmed. In summary, no adverse events related to the i.m. injection of bFGF with atelocollagen were observed over the follow-up period.

A clinical outcome survey regarding survival and major amputation was carried out after completion of the study protocol. The median period from injection to outcome survey in all patients was 478 days. Of the enrolled patients, 1 patient had major amputation of the target limb at 851 days after treatment, and there was no death after treatment (Figure 1).

\section{Secondary Endpoints}

Change in VAS score and 6-min walk distance after injection are shown in Figures 2,3. Median VAS improved markedly, from baseline (7.5 cm; IQR, 6.8-9.3), at 4 weeks (3.0 cm; IQR, 1.5-5.0; P<0.05), 24 weeks (0 cm; IQR, 0.0-0.5; $\mathrm{P}<0.05)$ to 48 weeks $(0 \mathrm{~cm}$; IQR, $0.0-0.6 ; \mathrm{P}<0.05$; Figure 2$)$. Seven patients became pain free in the follow-up period. Two patients trended to improve during the follow-up period, and 1 patient had exacerbated pain. These 3 patients, however, were not followed up sufficiently to 48 weeks after treatment. Change in VAS on a per-patient basis is shown in Figure S1.

In contrast, we excluded 2 patients from analyses of 6-min walk distance because they had a history of major amputation in the contralateral legs. The 6-min walk distance did not significantly improve, from baseline $(60 \mathrm{~m}$; IQR, 8.8-118), at 4 weeks (54 m; IQR, 8.8-123; $\mathrm{P}=0.89$ ), 24 weeks $(120 \mathrm{~m}$; IQR, 38-195; $\mathrm{P}=0.11)$ to 48 weeks $(174 \mathrm{~m}$; IQR, 36-274; $\mathrm{P}=0.18$; Figure 3).

The other efficacy parameters are listed on a per-patient basis in Table S1. The Fontaine and Rutherford classification in 4 out of 10 patients improved during the follow-up period. In 3 patients with ulcerous lesions, those in 2 patients reduced or healed during the follow-up period. In 2 patients with necrotic lesions, the areas of these lesion reduced during the follow-up period (from $1.84 \mathrm{~cm}^{2}$ to $0.02 \mathrm{~cm}^{2}$ and from $3.35 \mathrm{~cm}^{2}$ to $1.00 \mathrm{~cm}^{2}$ ). Cyanotic lesions disappeared in 2 patients at 4 weeks after injection.

\section{Discussion}

To the best of our knowledge, this is the first clinical trial evaluating the therapeutic effect of i.m. injection of bFGF with atelocollagen in patients with CLI (Fontaine classification III or IV). No serious procedure-related adverse events were detected, even in elderly patients, over the follow-up period. Additionally, there were no deaths and no incidence of major amputation in the 1 year after treatment.

The average 1-year limb amputation rate is approximately $30 \%$ in patients with CLI who are treated using conventional therapy, including bypass surgery and endovascular therapy. ${ }^{21}$ Moreover, the 1-year mortality risk for patients with CLI in 2015 remained very high at 16-36\%..22 By comparison, in the present study, the OS and MAF rates at 1 year after treatment were $100 \%$. Only 1 patient required major limb amputation at 851 days after treatment due to a relapse in ischemic pain at rest. No adverse events specifically related to the i.m. injection of bFGF with atelocollagen were observed over the follow-up period. Therefore, we suggest that the i.m. injection of bFGF with atelocollagen is safe for elderly patients with CLI.

With regard to therapeutic efficacy, VAS score improved significantly at 4, 24 and 48 weeks after treatment, and 7 patients became pain free during the follow-up period. The TACT trial reported the efficacy of BM-MNC implantation for no-option CLI patients. In the TACT trial, the VAS score and claudication distance significantly improved in no-option CLI patients with ASO or TAO. Although comparison is difficult due to the small number of cases in the present study, the improvement in VAS was similar to that in the TACT trial. Unlike the TACT trial, however, no improvement was obtained in 6-min walk distance. This might reflect the small patient number in this trial, the inclusion of older patients than in the TACT trial, and complications at baseline such as major amputation or compression fracture. Overall, the present findings support a therapeutic benefit of bFGF injection with atelocollagen solution on lower limb angiogenesis, with an acceptable level of efficacy. In addition, this treatment is less invasive than other cell therapies that have previously been reported for the treatment of CLI. Therefore, we speculate that the i.m. injection of bFGF with atelocollagen may become a feasible treatment for no-option CLI patients, especially those elderly patients with high cardiovascular risk. It will therefore be necessary to conduct a randomized controlled trial (RCT) to further evaluate the efficacy of this treatment for patients with CLI.

Therapeutic angiogenesis using bFGF with atelocollagen solution provides the following advantages: (1) sitespecific sustained delivery of bFGF without the use of cells, viruses or plasmids; (2) no requirement for invasive procedures, such as bone marrow harvesting; and (3) use of low serum bFGF, thereby reducing the risk of adverse drug reactions. Moreover, both atelocollagen and bFGF are already approved for use in clinical practice: atelocollagen, for soft-tissue augmentation; and bFGF, as a spray, for topical decubitus or skin ulcer healing. Several therapeutic approaches for angiogenesis (including VEGF, HGF, bFGF, and bone marrow cells) have previously been examined as potential treatment for CLI, with each treatment having its advantages and disadvantages. A direct injection of free growth factors previously failed to demonstrate efficacy in a clinical trial, ${ }^{14}$ presumably because the half-life of growth factors alone is too short to exert angiogenic effects in vivo. Gene transfer of growth factors has raised concerns owing to the unpredictable duration and level of gene expression, as well as the risk for immune or inflammatory responses to the genetic materials. Autologous bone-marrow cell transplantation requires an invasive procedure (the aspiration of cells under general anesthesia), while peripheral MNC transplantation requires the systemic treatment of granulocyte-colony stimulating factor, which may increase the risk for myocardial infarction.

The similar sustained release of bFGF from gelatin hydrogel microspheres has been examined in patients with CLI, ${ }^{15,16,23,24}$ as well as in other organs. ${ }^{25} \mathrm{~A}$ recent study by Kumagai et al showed the efficacy of the sustained release of bFGF using biodegradable gelatin hydrogel, with efficacy evaluated using transcutaneous oxygen pressure $\left(\mathrm{TcpO}_{2}\right)$, 6-min walk distance, pain at rest, and the cyanotic scale. ${ }^{16}$ They reported improvement in ischemic changes of the foot (ulcers), as well as improvements on the other measured outcomes, ${ }^{16}$ which were similar to 
those reported for bFGF-incorporated gelatin hydrogel. ${ }^{17}$ Gelatin hydrogel consists of microspheres that are difficult to inject, and is also more expensive than atelocollagen due to its complicated manufacturing process. Accordingly, the present method may be more preferable than gelatin hydrogel microspheres in terms of costs and convenience in practice.

In the present study, the i.m. injection of bFGF with atelocollagen was safe and effective for the treatment of CLI, especially that classed as Rutherford 4 or 5 . In addition, because this treatment was less invasive compared with conventional cell therapies, this treatment may be a new option for elderly patients with CLI. The evidence for this treatment, however, is not sufficient, and RCT are needed to evaluate the efficacy of this treatment in the future. Furthermore, in the next trial, we would like to increase the number of the enrolled patients and investigate the long-term outcomes of this treatment.

\section{Study Limitations}

This study had some limitations regarding the interpretation of trial results. First, the number of patients was relatively small and the follow-up period was limited. Given that currently approved cellular therapies for CLI (e.g., BM-MNC implantation) are applicable to patients $<80$ years old, many CLI patients $>80$ years old were enrolled in this study, leading to selection bias. Further larger and longer studies are needed to accurately evaluate the effects of treatment. Second, several efficacy parameters could not be evaluated in all patients, due to comorbidity and socioeconomic reasons, with 3 patients having insufficient evaluation of efficacy. In order to evaluate effectiveness more accurately, more stringent selection criteria are needed in the next trial. Third, accurate ABI data were not available for a few patients in the follow-up period, due to being unable to sit still due to severe pain. The ABI data were therefore not included in the present analysis. We can note, however, that in 6 patients presenting with abnormal $(<0.9)$ or unmeasurable ABI at baseline, ABI normalized in 3 patients during the follow-up period.

In addition, in this study, we did not evaluate some parameters of peripheral blood flow assessment such as skin perfusion pressure or $\mathrm{TcpO}_{2}$. These parameters therefore need to be evaluated in the next trial.

\section{Conclusions}

The i.m. injection of bFGF with atelocollagen was not associated with adverse events, and provided improvement in limb ischemic symptoms over the follow-up period. This may be a safe and feasible treatment for CLI. Future RCT are needed to evaluate the efficacy of the i.m. injection of bFGF with atelocollagen.

\section{Acknowledgments}

The authors thank Ms. Kurisu for data management, Ms. Ogawa and Mr. Sakamine for source data verification, and Dr. Fukushima, Dr. Kojima, Dr Kagimura, Dr. Kaneda, Dr. Nagai, Dr. Kikuchi, Mr. Nakatani, Ms. Oh, and Mr. Yoshidomi for critically reading the manuscript and providing useful suggestions.

\section{Disclosures}

Kyoto University, Kyoto Prefectural Public University Corporation, Kaken Pharmaceutical Co., Ltd., and Koken Co. Ltd., are the assignees of patent "Sustained-release Pharmaceutical Composition" (PCT/WO/2012/050184_A1. Patent filed 14 October 2011). Dr.
Fukushima M. and Dr. Matoba S. are two of the inventors. The other authors declare no conflicts of interest.

\section{References}

1. Anderson JL, Halperin JL, Albert NM, Bozkurt B, Brindis RG, Curtis LH, et al. Management of patients with peripheral artery disease (compilation of 2005 and 2011 ACCF/AHA guideline recommendations): A report of the American College of Cardiology Foundation/American Heart Association Task Force on Practice Guidelines. Circulation 2013; 127: 1425-1443.

2. Berger JS, Hiatt WR. Medical therapy in peripheral artery disease. Circulation 2012; 126: 491-500.

3. Wennberg PW. Approach to the patient with peripheral arterial disease. Circulation 2013; 128: 2241-2250.

4. Fujita Y, Kinoshita M, Furukawa Y, Nagano T, Hashimoto H, Hirami Y, et al. Phase II clinical trial of CD34+ cell therapy to explore endpoint selection and timing in patients with critical limb ischemia. Circ $J$ 2014; 78: 490-501.

5. Kawamoto A, Katayama M, Handa N, Kinoshita M, Takano $\mathrm{H}$, Horii $\mathrm{M}$, et al. Intramuscular transplantation of G-CSFmobilized CD34(+) cells in patients with critical limb ischemia: A phase I/IIa, multicenter, single-blinded, dose-escalation clinical trial. Stem Cells 2009; 27: 2857-2864.

6. Kinoshita M, Fujita Y, Katayama M, Baba R, Shibakawa M, Yoshikawa K, et al. Long-term clinical outcome after intramuscular transplantation of granulocyte colony stimulating factormobilized CD34 positive cells in patients with critical limb ischemia. Atherosclerosis 2012; 224: 440-445.

7. Onodera R, Teramukai S, Tanaka S, Kojima S, Horie T, Matoba $\mathrm{S}$, et al. Long-term clinical outcomes for patients with lower limb ischemia implanted with G-CSF-mobilized autologous peripheral blood mononuclear cells. Atherosclerosis 2010; 208: 461 -466.

8. Matoba S, Tatsumi T, Murohara T, Imaizumi T, Katsuda Y, Ito $\mathrm{M}$, et al. Long-term clinical outcome after intramuscular implantation of bone marrow mononuclear cells (Therapeutic Angiogenesis by Cell Transplantation [TACT] trial) in patients with chronic limb ischemia. Am Heart J 2008; 156: 1010-1018.

9. Onodera R, Teramukai S, Tanaka S, Kojima S, Horie T, Matoba $\mathrm{S}$, et al. Bone marrow mononuclear cells versus G-CSF-mobilized peripheral blood mononuclear cells for treatment of lower limb ASO: Pooled analysis for long-term prognosis. Bone Marrow Transplant 2011; 46: 278-284.

10. Idei N, Soga J, Hata T, Fujii Y, Fujimura N, Mikami S, et al. Autologous bone-marrow mononuclear cell implantation reduces long-term major amputation risk in patients with critical limb ischemia: A comparison of atherosclerotic peripheral arterial disease and Buerger disease. Circ Cardiovasc Interv 2011; 4: 15-25.

11. Kondo K, Yanishi K, Murohara T, Matoba S, Katsuda Y, Ito $\mathrm{M}$, et al. Long-term clinical outcomes survey of bone marrowderived cell therapy in critical limb ischemia in Japan. Circ J 2018; 82: $1168-1178$.

12. Lee K, Silva EA, Mooney DJ. Growth factor delivery-based tissue engineering: General approaches and a review of recent developments. J R Soc Interface 2011; 8: 153-170.

13. Ouma GO, Jonas RA, Usman MH, Mohler ER. Targets and delivery methods for therapeutic angiogenesis in peripheral artery disease. Vasc Med 2012; 17: 174-192.

14. van Royen N, Piek JJ, Schaper W, Fulton WF. A critical review of clinical arteriogenesis research. J Am Coll Cardiol 2009; 55: $17-25$.

15. Marui A, Tabata Y, Kojima S, Yamamoto M, Tambara K, Nishina T, et al. Sustained-release vancomycin sheet may help to prevent prosthetic graft methicillin-resistant Staphylococcus aureus infection. Circ J 2007; 71: 1181-1186.

16. Kumagai M, Marui A, Tabata Y, Takeda T, Yamamoto M, Yonezawa A, et al. Safety and efficacy of sustained release of basic fibroblast growth factor using gelatin hydrogel in patients with critical limb ischemia. Heart Vessels 2016; 31: 713-721.

17. Sano A, Maeda M, Nagahara S, Ochiya T, Honma K, Itoh H, et al. Atelocollagen for protein and gene delivery. Adv Drug Deliv Rev 2003; 55: 1651-1677.

18. Fukushima M, Matsubara H, Matoba S, inventors; Kyoto Univ., Kyoto Prefectural Public University Corporation, Kaken Pharmaceutical Co., Ltd. Koken Co,. Ltd., assignee. Patent PCT/JP2011/073632. 2011; October 14. WO2012/050184.

19. Olin JW. Thromboangiitis obliterans. N Engl J Med 2000; 343: 864-869.

20. Shionoya S. Diagnostic criteria of Buerger's disease. Int J Cardiol 1998; 66: S243-S245. 
21. Norgren L, Hiatt WR, Dormandy JA, Nehler MR, Harris KA, Fowkes FG, et al. Inter-society consensus for the management of peripheral arterial disease (TASC II). J Vasc Surg 2007; 45: S5-S67.

22. Reinecke H, Unrath M, Freisinger E, Bunzemeier H, Meyborg M, Luders F, et al. Peripheral arterial disease and critical limb ischaemia: Still poor outcomes and lack of guideline adherence. Eur Heart J 2015; 36: 932-938.

23. Hashimoto T, Koyama H, Miyata T, Hosaka A, Tabata Y, Takato T, et al. Selective and sustained delivery of basic fibroblast growth factor (bFGF) for treatment of peripheral arterial disease: Results of a phase I trial. Eur J Vasc Endovasc Surg 2009; 38: $71-75$.

24. Ito N, Saito S, Yamada MH, Koizuka S, Obata H, Nishikawa $\mathrm{K}$, et al. A novel bFGF-GH injection therapy for two patients with severe ischemic limb pain. J Anesth 2008; 22: 449-452.
25. Hakuba N, Tabata Y, Hato N, Fujiwara T, Gyo K. Gelatin hydrogel with basic fibroblast growth factor for tympanic membrane regeneration. Otol Neurotol 2014; 35: 540-544.

\section{Supplementary Files}

\section{Supplementary File 1}

Figure S1. Per-patient change in visual analogue scale (VAS) score, after i.m. injection of basic fibroblast growth factor with atelocollagen, in patients with critical limb ischemia.

Table S1. Subject-specific baseline characteristics and clinical outcomes

Please find supplementary file(s);

http://dx.doi.org/10.1253/circj.CJ-18-0815 